EXEMPLARIa Classica

Journal of Classical Philology

16, 2012, pp. 137-147

ISSN 1699-3225

\title{
COLACIÓN DEL IBIS DE OVIDIO EN EL MS. BEROL. DEUTSCHE STAATSBIBL. DIEZ. B SANT. 4
}

\author{
JosÉ M. García HeRnÁNDEZ \\ Grupo de Investigación "Nicolaus Heinsius" \\ jmg.hernandez@yahoo.es
}

SUMMARY

We introduce the collation of the ms.

Berol. Deutsche Staatsbibl. Diez. B.

Sant. 4, which contains several interesting lectures. Our collation shows that this ms. has been erroneously identified with the ms. Berolinensis Crevennae collated by R. Merkel in 1837.

KEYWORDS

Ovid, Ibis, Berol. Deutsche Staatsbibl.

Diez. B. Sant. 4, Berolinensis Crevennae
RESUMEN

Presentamos la colación del ms. Berol. Deutsche Staatsbibl. Diez. B. Sant. 4, que contiene varias lecturas interesantes. Nuestra colación muestra que este ms. ha sido erróneamente identificado con el ms. Berolinensis Crevennae colacionado por R. Merkel en 1837.

Palabras Clave

Ovidio, Ibis, Berol. Deutsche Staatsbibl. Diez. B. Sant. 4, Berolinensis Crevennae

Fecha de recepción: 19/06/2011

Fecha de aceptación y versión final: 30/08/2012

1. El Berolinensis Deutsche StaAtsbibliothek Diez. B Sant. 4.

El manuscrito ${ }^{2}$ contiene una miscelánea de autores en la que encontramos gran parte de la obra de Ovidio a excepción de Metamorfosis, Amores y Fastos. Se trata de un códice en papel con 187 folios de 265 x 200 mm., de los

${ }^{1}$ Este trabajo ha sido realizado en el marco del Proyecto de Investigación HUM-4534 de la Consejería de Innovación, Ciencia y Empresa de la Junta de Andalucía. Queremos agradecer las sugerencias de los informantes anónimos de Exemplaria Classica, así como las de los profesores Antonio Ramírez de Verger y Luis Rivero García, el doctor José Antonio Bellido Díaz y mi colega Samuel Díez Reboso.

${ }^{2}$ Hemos realizado la colación a partir de una reproducción digital de este manuscrito, que en adelante será designado por la negrita cursiva $\boldsymbol{B}$. En relación al mismo, uid. Ursula Winter, Die europäischen Handschriften der Bibliothek Diez: Teil 1. Die Manuscripta Dieziana B Santeniana, Teil 2. Die Libri impressi cum notis manuscriptis. Leipzig 1986, 18-22. Puede consultarse on-line en el siguiente enlace: http://www.manuscripta-mediaevalia.de/hs/ katalogseiten/HSK0479_b018_JPG.htm. 
siglos XIV y XV, si bien todas las filigranas apuntan a Italia y al siglo XIV. Tiene una caja de escritura de un promedio de $220 \times 160 \mathrm{~mm}$. en la que el texto se distribuye en dos columnas de 42 líneas cada una, excepto los ff. $11^{\mathrm{v}}-2^{\mathrm{r}}$ y $169^{r}-87^{v}$. No presenta línea de escritura y la división está limitada por líneas ciegas. Cambia de mano en el folio $169^{\mathrm{r}}$ hasta el folio $187^{\mathrm{v}}$, donde presenta letra bastarda. Las glosas marginales e interlineales son esporádicas, dándose la circunstancia de que se encuentran glosas en francés. Casi sin adornos en las capitales iniciales, se produce la desaparición de las mismas a partir del folio $169^{r}$. La portada del manuscrito se ha perdido en su mayor parte debido a un corte. Presenta tres tipos de numeración moderna en el ángulo superior derecho de cada recto. La parte que contiene Ibis, folios $145^{\text {va }} 9^{\text {ra }}$, presenta una media de entre 80-4 versos distribuidos en dos columnas de 40 a 42 versos cada una. Los versos 579-626 se han perdido, de modo que el verso 578 da fin al folio $148^{\mathrm{vb}}$ y el 627 da comienzo al folio $149^{\text {ra }}$. Hay una imperfección en el folio $149^{r}$ que provocó que el copista tuviera que adaptar el tamaño de los versos 636-43 al borde de la misma. Se lee con claridad un incipit (Ovidius de in Ibim) así como el explicit (explicit Ovidius in Ibim). Según Winter ${ }^{3}$, este manuscrito fue colacionado por Merkel para su edición de Tristia e Ibis de 1837 bajo el nombre de Berolinensis Crevennae, del cual dice La Penna que se hallaba extraviado o perdido y que por eso no pudo inspeccionarlo para su edición de $1957^{4}$.

En cualquier caso, después de comparar nuestra colación con la realizada por Merkel para su citada edición, hemos de decir que cabe la duda de que este manuscrito sea el Codex Crevennae o Berolinensis Crevennae empleado por dicho autor ${ }^{5}$, pues hemos constatado la existencia de muchísimas variantes entre ambas colaciones, he aquí sólo algunos ejemplos ${ }^{6}$ :

$\mathbf{1}$ bis iam mihi] iam bis modo $\mathbf{B}$ modo iam bis $\boldsymbol{l} 16$ vivi] miseri $\mathbf{B}$ vivi $\boldsymbol{l} \quad 64$ sic vita series] sic vite series $B$ sit acta tuae $\boldsymbol{l} \quad 68$ cum Iove] cum Iane B cum Iove $\boldsymbol{l} \mathbf{9 3}$ fictum] fixum B fictum $\boldsymbol{l} 101$ ominibusque] hominibusque $\mathbf{B}$ ominibusque $\boldsymbol{l} 107$ amnis] animas B oannas $l \quad 157$ ante os] animos B ante os $l(155) \quad 186$ tertia] tertius B tertia $\boldsymbol{l}(184) \quad 273 \mathrm{ut}]$ ubi B ut $\boldsymbol{l}(271) \quad 291$ parum

${ }^{3}$ Winter, Die europäischen Handschriften, 20-1.

${ }^{4}$ A. La Penna, Publi Ovidi Nasonis. Ibis, Firenze 1957, cxlvii.

${ }^{5}$ Vid. la descripción que de este manuscrito realiza R. Merkel en P. Ovidii Nasonis Tristium libri quinque et Ibis, Berolini 1837, 34, núm. 15 y 408, núm. 18.

${ }^{6}$ Daremos en primer lugar la lectura correcta según el texto establecido por La Penna en 1957, luego la de nuestro manuscrito bajo la sigla $\mathbf{B}$, por último la lectura que propone Merkel para el Crevennae bajo la sigla $\boldsymbol{l}$, que es la que él emplea en su edición. Téngase en cuenta que La Penna conserva el dístico 133-4 tras 132, mientras que Merkel lo excluye, por lo que en la edición de éste último deben restarse dos versos respecto de la edición de La Penna a partir del verso 132. En cualquier caso, para facilitar la localización de los versos, anotamos la numeración de Merkel entre paréntesis. 
mitis] parum mittis B parum mutis $\boldsymbol{l}(289) \quad 336$ Actea] athea B Aactaea $\boldsymbol{l}$ (334) Post 342 versus 343 sequitur $\mathbf{B}$, sed ait Merk. (340): "post hunc versum $\boldsymbol{l}$ denuo habet vv. 635-6" (637-8 La Penna) 407 Polypemone] polihemane B politemone $\boldsymbol{l}$ (405) 422 subtrahitur] subiceretur B subiiceret $l(420) \quad 443$ caeni] scevi B caeni $\boldsymbol{l}$ (421) $\mathbf{4 5 8}$ ut est celeri victaque] et ut sceleri victima B ut est celeri victaque $\boldsymbol{l}(456) \quad \mathbf{5 2 6}$ exitii] excicii B exitii $\boldsymbol{l}(\mathbf{5 2 4})$ 563 videras quod] videras qui B videras quod $\boldsymbol{l}(561) \quad 630$ necis] meus B necis $\boldsymbol{l}(628)$

Sin embargo, la existencia de estas variantes de lectura no es impedimento para que compartan las mismas omisiones de versos, así como ciertos errores comunes, de los que presentamos a continuación algunos ejemplos ${ }^{7}$ :

20 hic] is 30 ei] e Dist. 53-4 om. 91 quasque] quas 101 laevis] undis 124 optatam fugiat] optatumque fugiet 127 evenient] et venient Dist. 143-4 deest 150 sive] inque 182 praebet] prestat 192 nunc] et 210 stella levisve] stellaque clara 237 stipite] cespite 247 ille] hic 292 fixus alas] pasce tuo 294 proiciare] precipitare $\mathbf{3 0 8}$ Cantharidum] tassalidum $\mathbf{3 6 1}$ neve magis pia sit capiti parentis amica] filia neve magis capiti sit fida parentis $\quad v v .362-3 \mathrm{om} .403$ vindice] vertice 409 pressas] pransas Dist. 439-40 scriptum post vv. 338, 637-8 et deinde geminatum post 438439 prius ense resecta] prius ore resecta 453 Cybeleia] tibeia Dist. $461-2$ abest post $460 \quad 463$ Cycneius heros] lingneus heror B linguens heros $\boldsymbol{l} \mathbf{4 8 8}$ iuvit] iuit $v \boldsymbol{v}$. 530-1 absunt $\quad v v$. 579-626 absunt $\quad$ vv. 633-4 desunt

Aunque serán necesarias nuevas investigaciones que engloben el resto de las obras transmitidas en ambos manuscritos para terminar de aclarar este punto, parece que las variantes de lectura y los errores comunes que hemos constatado nos llevan a dos conclusiones: 1 . $\boldsymbol{B}$ y $\boldsymbol{l}$ son manuscritos diferentes; 2. ambos provienen de un mismo exemplar, hasta ahora perdido.

${ }^{7}$ Como dijimos en la nota 5 , réstense dos versos para la edición de Merkel a partir del verso 132. Coinciden todas las omisiones excepto la del verso 168 contenida en $\boldsymbol{B}$ (que no se aprecia en $\boldsymbol{l}$ ) y, aunque no es una omisión, el despiste cometido por el copista del Crevennae, que escribe el dístico 637-8 tras 338, y luego lo repite tras 342 (error no contenido en $\boldsymbol{B}$ ). 


\section{EL ORDEN DE LOS VERSOS EN $\boldsymbol{B}^{8}$}

Como en todos los manuscritos conservados, el orden de los versos presenta en $\boldsymbol{B}$ una disposición irregular:

1-38, 133-4, 39-52, 53-4 omissit., 119, 122-121-120, 123-30, $130 \mathrm{ab}^{9}, 131-2,133-4,135-42,143-4$ om., 145-67, 168 om, 169338, 637-8, 439-40, 461-2, 339-61, 362-3 om., 364-438, 439-40, 441-60, 463-529, 530-1 om., 532-47, 548 om., 549-78, 579-626 om., 627-32, 633-4 om., 635-6, 637-8, 639-44.

Se observa que el copista comete muchos errores de omisión, siendo el mayor de ellos el que se produce en el fragmento 579-626, una laguna en el texto de 48 versos. Si tomamos en consideración el número de versos por hoja que se reproducen en $\boldsymbol{B}$, no es posible en principio que se haya perdido un folio, pues el número de versos perdidos no cuadra con el número de versos por folio de nuestro manuscrito. Dicha pérdida, sin embargo, sí puede ser achacable al exemplar del que proceden $\boldsymbol{B}$ y $\boldsymbol{l}$, en el que con toda seguridad el copista se saltó el folio que contenía estos versos.

Por otra parte, tras cotejar este orden con el de los códices más importantes, se obtiene que el códice $\boldsymbol{B}$ comparte con $\mathrm{T}$ y con los manuscritos pertenecientes a la tercera familia el rasgo de situar el dístico 133-4 al comienzo del poema, si bien TVH lo hacen tras el verso $40, \mathrm{~A}$ tras el $43, \mathrm{Z}$ tras el $44, \mathrm{~F}$ en la parte superior de la hoja, en una sola línea sobre el verso 39 , mientras que $\boldsymbol{B}$ lo inserta tras el verso 38 , adelantando de este modo su posición. Por

\footnotetext{
${ }^{8}$ Creemos oportuno presentar en esta nota el orden de versos de los potiores pertenecientes a cada una de las familias tal como lo describe La Penna, Publi Ovidis Nasonis, Lxxvii. $1^{a}$ familia G: 1-40, 43-130, Distichon interpolatum, 131-2 om., 133-338, 637-8, 639-44. / P:1-40, 43-130, Dist. interp., 131-2 habet, 133-338, 637-8, 639-44. / $\mathrm{P}_{1}: 1-40,43-130$, Dist. interp., 131338, 339-571, 572-3 om., 574.636, 636-44. / E: 1-40, 43-130, Dist. interp. ex 461-2 conflatum, 339-636, 637-8 om. (sed 637-8, 461-2 in marg. ad 338 scripsit manus scholiorum), 639-44./ $2^{a}$ familia T: 1-40, 133-4, 43-130, Dist. interp., 131-2, 133-4, 135-338, 439-40, 461-2, 339-438, 441-60, 463-636, 637-8, 639-44. / $3^{a}$ familia F: 1-40, 133-4 (adscripti sunt in initio paginae quae a $v .39$ incipit, sed, cum in initio prioris columnae non satis esset spatii, 134 in initio alterius columnae scriptus est), 43-130, 131-2, Dist. interp., 133-4, 135-338, 637-8, 439-40, 461-2, 339-438, 441-60, 463-636, 639-44. / V: 1-40, 133-4, 43-130, 131-2, Dist. interp., 133-4 om., 135-338, 637-8, 439-40, 461-2, 339-414, 417-8, 415-6, 419-38, 441-60, 463-636, 639-44. / H: 1-40, 133-4, 43-130, Dist. interp., 131-288, 291-2, 289-90, 293-338, 637-8, 439-40, 461-2, 339-438, 441-60, 463-636, 639-44. /A: 1-40, 43, 133-4, 44-130, 131-2, Dist. interp., 133-4, 135-338, 637-8, 439-440, 461-2, 339-438, 441-60, 463-506, 509-510, 507-8, 511-636, 639-44. / Z:1-40, 43-4, 133-4, 45-130, Dist. interp., 131-262, 263-4 om., 265-338, 637-8, 439-40, 461-2, 339-438, 439-40, 441-60, 461-2, 463-506, 509-10, 507-8, 511-636, 639-44.

${ }^{9}$ Denominamos así al dístico Finiet illa dies que te mihi subtrahit olim / Finiet illa dies que mihi (tibi en $\boldsymbol{B}$ y G) tarda venit. Con esta numeración seguimos a Silvio M. Medaglia en "Riflessioni sul testo dell'Ibis di Ovidio (I)", Tradizione, ecdotica, esegesi. Miscellanea di studi, Napoli 2006, 137, quien además considera que este dístico es genuino del Ibis en lugar de espurio, como ha venido considerando la totalidad de los editores.
} 
otro lado, tras el verso 119 ha recolocado los versos 122, 121 y 120 en este orden como producto de un despiste. Tras 130 presenta el dístico 130ab que se encuentra en todos los códices conocidos (excepto en $\mathbf{o}^{10}$ ) y que los editores prefieren eliminar del texto, si bien R. Guarino lo mantiene entre corchetes por atención a la tradición manuscrita más que a las conjeturas humanísticas ${ }^{11}$. Tras 132 repite el dístico 133-4 y entre los versos 338-9 encontramos tres dísticos interpolados: 637-8, 439-40, 461-2, que de nuevo muestran un perfecto paralelismo con todos los códices potiores que integran la tercera familia: FVHAZ, pero con la particularidad, no registrada en estos códices (excepto en $\boldsymbol{Z}$ ), de que $\boldsymbol{B}$ repite los versos 637-8 y 439-40 tras 636 y 438 respectivamente. Las interpolaciones y desplazamientos de versos nos permiten, por tanto, situar este manuscrito en la órbita de la tercera familia, más concretamente de $\mathrm{H}$, como veremos luego.

\section{Posición STEMMATICA DE $\boldsymbol{B}$ Y VALORACIÓN DE SUS LECTURAS}

El manuscrito $\boldsymbol{B}$ comparte con el arquetipo medieval ( $\omega$ ) todos los errores que para éste señaló La Penna, Publi Ovidi Nasonis LXxxviII ${ }^{12}$, sin que hayamos observado que lo corrija en ninguno de sus puntos:

84 Chao] chori $\boldsymbol{\omega}$ thori B 178 Aegypti] egisti 229 inbuerunt] imbuerant 256 inermis opem] inerme potens $\omega$ inherme potens B 323 Alevas] alebas $\mathbf{3 4 8}$ Callirhoesque] cal(1l)igonesque $\boldsymbol{\omega}$ caliganesque B 378 Sphinga] spina B spinga G spy(i)nga cett. 412 Cercyonea] geri(y)onea 419 et] ut 447 Pytheides] pent(th) ides 502 Phalaeceae] paphegee $P$ paphagee $\mathrm{GP}_{1} \mathrm{TZ}$ pefagee $\mathrm{F}$ pesagee $\mathrm{V}$ pegasee $\mathrm{HA}$ phephigee $\mathrm{B} \quad \mathbf{5 1 2}$ Leoprepidae] leporide $\mathrm{G}$ leo tepide $\mathbf{P}_{1}$ leotrepido $\mathbf{P}$ leotoride $\mathbf{F Z}$ li $(\mathrm{y})$ coride TVHA lecoride BE 513 Evenus] ebenus $\mathrm{BPP}_{1}$ EFA hebenus $\mathrm{GT}$ habenus $\mathrm{V}$ ebennus $\mathrm{Z}$ ebeneus $\mathrm{H} \quad \mathbf{5 1 5}$ Astacidaeque] i(hi)rtacideque EFHB Tacideque G Ytacideque $\mathbf{P}$ Hyrchadieque $P_{1} \quad 523$ Athenin] Athenas

Además de los errores que comparte con el arquetipo, en $\boldsymbol{B}$ se encuentran otros que aparecen de forma simultánea en distintos mss. pertenecientes a cada una de las familias:

16 vivi] miseri $P_{1}$ EyoTFVHAZx 36 quem] quam GPPEOTFVHAZVH 76 netis] nectis $\mathrm{GPP}_{1} \mathrm{~V}_{1}$ oFHAZ 84 Chao] cori $G$ chori $\mathrm{PP}_{1} \mathrm{EV}_{1}$ TFVAZ thori $\mathrm{BHx} \quad 85$ canuntur] canantur $\mathrm{EV}_{1} \mathrm{yH} \quad 86$ peragunt] peragant $\mathrm{P}_{1} \mathrm{EH} \quad 141$ tunc] tu

\footnotetext{
${ }^{10}$ Para el orden de versos de este códice y su descripción véase La Penna, Ibis, cxxxi.

${ }^{11} \mathrm{R}$. Guarino, Los comentarios al Ibis de Ovidio, Frankfurt am Main 1999, 37.

${ }^{12}$ Excepto el error del verso 603, que en B se encuentra omitido.
} 
$\mathrm{P}_{1} \mathrm{~V} 178$ Aegypti] egisti $\mathrm{B}$ egesti $\mathrm{H}$ aegisti GPP $\mathrm{EV}_{1}$ TFVHAZ 256 armatique] armatusque $\mathrm{GP}_{1} \mathrm{EV}_{1}$ oTFVHAZ 265 ille] ipse $\mathrm{PP}_{1} \mathrm{~V}_{1}$ oTFVAZ 323 Alevas] alebas GPP EyTVHAZx 347 Oetaeo] acteo PyTZ 348 Cal(1)irhoesque] caliganesque B cal(1) igonesque GPPEoTFHVZ 419 et] ut GPPEoyTFHAVZ 429 solis] rursus PEyTA 430 rursus] solis $\mathrm{PP1EV}_{1}$ TVHAZ 466 Theudotus] theodorus $\mathrm{BGV}_{1} \mathrm{yx}$ theodotus oTVAZ $\mathbf{4 8 3}$ Hypsipyles] y(hy)siphiles BPETHAZ 499 Lindia] lidia BETVZ lydia $V_{1} \quad 507$ atque] sive EFVHZ $\mathbf{5 3 9}$ tardae] tarde EoFVH $\mathbf{5 4 5}$ Harpagides] arpagie $\mathbf{B}$ arphagie TH $\mathbf{5 5 9}$ Anyti] veluti GFVHZ $\mathbf{5 6 4}$ in urbe] ab arce $\mathrm{V}_{1} \mathrm{yHZ} \mathbf{5 7 3}$ Psamathes] samatis yA

Por otro lado, $\boldsymbol{B}$ comparte con todos los potiores de la primera y la tercera familias algunos errores que hasta hoy habían sido considerados únicos de ciertos manuscritos pertenecientes a ellas o exclusivos de una $u$ otra:

20 hic] is y 25 meritas grates] magnas grates $H \quad \mathbf{3 0}$ qua $]$ quam $P$ 32 lumina] tempora $\mathrm{H} \quad 48$ calfacti] calefacti $\mathrm{A} \quad \mathbf{8 9}$ ut non] non ut Z 90 Pasiphaes] phasiphes y 101 omnibusque] hominibusque $\mathrm{P} 127$ Evenient] et venient $\mathrm{V}_{1} \quad 197 \mathrm{ab}$ Ida] in hibla $\mathbf{B}$ in ibla $\mathrm{H}$ 210 stella levisve] stellaque clara $\mathrm{H} \quad 229$ inbuerunt] imbuerant GPP Eyo 241 movimus] vovimus yo 247 ex me] et me o 276 cui] qui $\mathrm{H} \quad 294$ proiciare] precipitare y 299 Achei] athei $\mathrm{P}$ 301 Achillidem] achilleidem FVHZ 302 tegula] ragula A 313 parantes] parentes $\mathrm{P}_{1}$ Eo 319 Atarnites] acarnides E insutus] inductus y 321 Pharaei] philei y 322 leto] lecto y 324 experiare] experiere $\mathrm{H} \quad \mathbf{3 4 4}$ qui] cui FAZ habet] erat FAZ $\mathbf{3 8 8}$ ut] aut FVHA 395 Lemnia] lennia $V_{1} \quad 404$ Nessus] nesi $P_{1} E^{a c}$ 415 Aetna] o(ho)ra GPP Eoy 418 pontem] pontum $\mathrm{P}_{1} \mathrm{~V}_{1} \quad 439$ ense] ore $\mathrm{G} \quad 443$ caeni] scevi $\mathrm{E} \quad \mathbf{4 4 6}$ iactis] aparsis $V_{1} \quad \mathbf{4 5 2}$ culter] cultor $\mathrm{V} \quad \mathbf{4 5 7}$ subito... magnae] magnae... subito $\mathrm{PP}_{1} \mathrm{EV}_{\text {. }}$ 494 socraticum] sacraticum $Z \quad \mathbf{5 2 6}$ exitii] excicii $G \quad \mathbf{5 2 7}$ Oresti] oreste o 533 silva] silvis FHA $\mathbf{5 4 1}$ Achaeo] achevo o $\mathbf{5 4 3}$ saxis] flammis VH $\mathbf{5 4 9}$ utve] utque VHZ $\mathbf{5 5 1}$ nudave] nudaque FVHAZ 635 utque] utve FHAZ

Estos errores, en conjunción con el orden de versos, nos hacen colegir que el ms. $\boldsymbol{B}$ presenta un alto grado de contaminación con la primera familia, pero además permiten emparentar $\boldsymbol{B}$ con los manuscritos de la tercera, concretamente con el grupo FVH, como muestra la presencia de ciertos errores comunes, de los que mostramos a continuación algunos ejemplos: 
301 Achillidem] achilleidem FVHZ 388 ut] aut FVHA 533 silva] silvis FHA $\mathbf{5 4 9}$ utve] utque $\mathrm{VHZ} \mathbf{5 5 1}$ nudave] nudaque FVHAZ 635 utque] utve FHAZ

Pero sobre todo $\boldsymbol{B}$ muestra una especial relación con $\mathrm{H}$ como parecen atestiguar reveladores errores comunes:

\section{5 magnas grates $\mathbf{B H} \quad 32$ tempora $\mathbf{B H} \quad 197$ in hibla $\mathbf{B}$ in ibla $\mathrm{H}$ 210 stellaque clara $\mathrm{BH}$}

Por otra parte, $\boldsymbol{B}$ presenta una gran cantidad de lecturas únicas, algunas de las cuales son las siguientes:

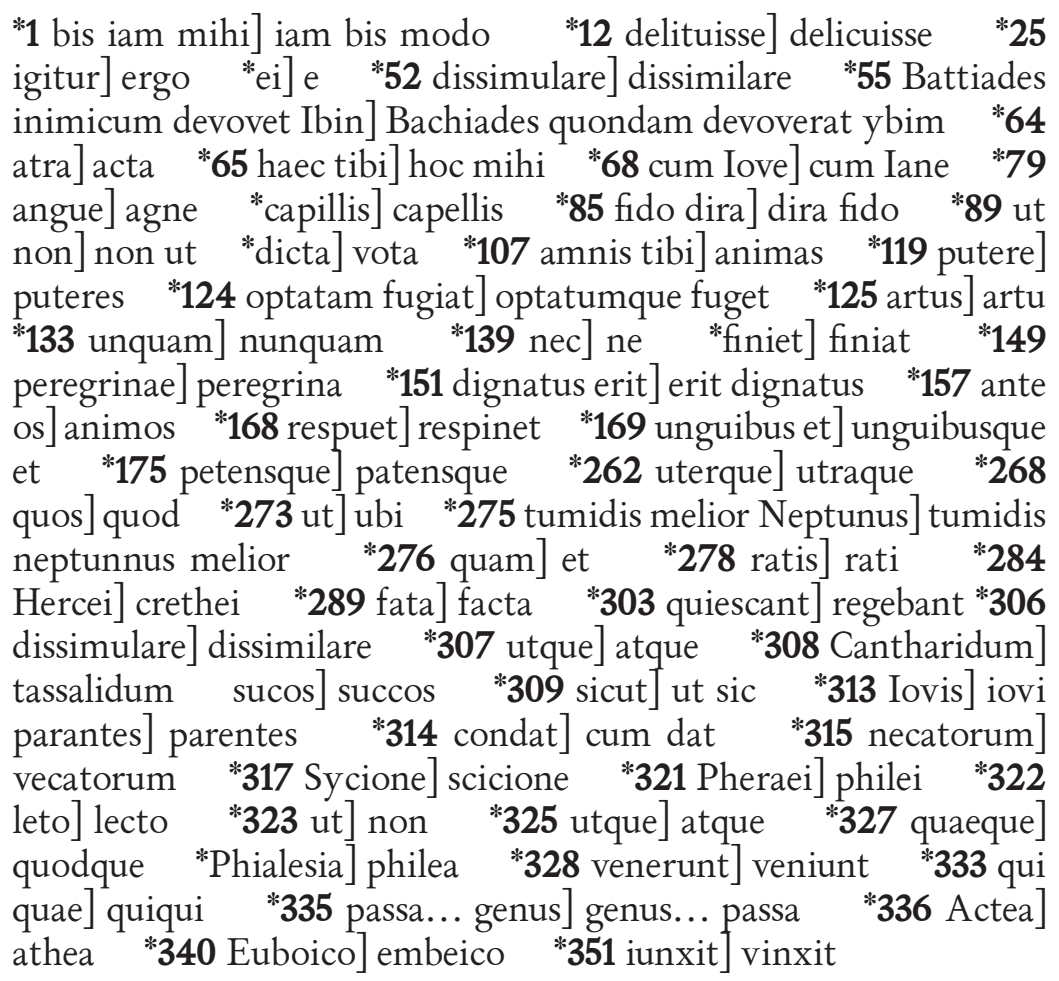

La inmensa mayoría de estas lecturas únicas son errores, pues algunas atentan contra la métrica más elemental, otras se deben a fallos de interpretación de abreviaturas y otras contienen soluciones que semánticamente no cuadran con el contexto en el que se encuentran.

Todos los errores que acabamos de mostrar, junto con estas lecturas únicas, nos hacen concluir que, como ya dijimos arriba, $\boldsymbol{B}$ es un manuscrito muy 
contaminado. Sin embargo no puede desecharse por completo, pues presenta en ciertos pasajes algunas lecturas interesantes ${ }^{13}$ :

55 Nunc quo Bachiades quondam devoverat ybim 89 Quaeque precor fiant ut non mea vota sed illa 175 Sisyphus est illic saxum volvensque patensque 192 Versabunt celeres et nova membra rotae ${ }^{14}$ 207 Illae me lacrimis facient sine fine beatum ${ }^{15} \quad$ 365-366 ut iuvenes pereas proiecta cadavera quorum / oraque Pisaeae sustinuere fores 352 aequoris aspectans huius et huius aquas 414 evenient iustis non leviora malis 420 Destituasque tuas usque petitas oves ${ }^{16}$

A todas estas lecturas habría que añadir una muy particular que $\boldsymbol{B}$ comparte con el ms. $\boldsymbol{G}$; hablamos de la variante tibi que se registra en el pentámetro del dístico 130ab que Medaglia considera genuino del texto de Ibis en lugar de espurio:

finiet illa dies quae te mihi subtrahet olim

finiet illa dies quae mihi (tibi BG) tarda venit.

El filólogo italiano considera correcta la lectura de $\mathrm{G}$ en tanto que semánticamente es tibi la palabra que mejor encaja en este pasaje, mientras que mihi pudo haberse transmitido por error en el resto de los códices debido a la proximidad de los versos 130 b y $132^{17}$. En este sentido, sólo $\mathbf{G}$ y $\boldsymbol{B}$ habrían transmitido la lectura correcta.

\section{CONCLUSIÓN}

Hemos presentado la colación del ms. Berol. Deutsche Staatsbibl. Diez. B. Sant. 4, que en contra de la opinión de Ursula Winter no debe identificarse con el Berolinensis Crevennae empleado por Merkel para su edición de Tristia e Ibis de 1837. De sus lecturas se desprende que es un manuscrito muy contaminado pero que en algunos pasajes, sin embargo, ha transmitido lecturas

${ }^{13}$ Se destaca en cursiva la lectura del ms. $\boldsymbol{B}$.

${ }^{14} E t$ en lugar de nunc, posiblemente la lectura correcta, pues mejora la transición del castigo de Sísifo al de Ixión, condenado por Zeus a dar vueltas atado con serpientes a una rueda ardiendo, Cf, Ov., Met. 4.461 y ss.

${ }^{15}$ Illae en referencia a ruinae (v. 205). Que te ocurran tantas desgracias que hasta yo derrame lágrimas por ti. Aquéllas me harán feliz a pesar de mis lágrimas.

${ }^{16}$ El interés de esta lectura no está en la literalidad de la misma, sino en el hecho de que permite deducir una nueva a partir de ella: Destituatque tuas usque petitas opes. Posiblemente la lectura correcta, en tanto que se refiere al hecho de que Ibis se vea privado de las riquezas a que aspira, lo cual casa a la perfección con la alusión a Pluto del verso anterior, al tiempo que enlaza con la situación del propio Ovidio, para el que Ibis había pedido la publicatio bonorum con intención de apropiarse de sus bienes.

${ }^{17} 130 \mathrm{~b}$... quae mihi tarda venit / 132 ... quae mihi sera venit. 
dignas de ser tenidas en cuenta, por lo que consideramos que, a pesar de su alto grado de contaminación, tiene cierto valor para la constitutio textus.

\section{COLACIÓN DEL MANUSCRITO $\boldsymbol{B}$}

*1 bis iam mihi] iam bis modo 2 inerme inherme $^{18} \quad$ *12 delituisse] delicuisse 16 vivi] miseri 17 quassa] cassa 20 hic] is 23 est] om. *25 igitur] ergo meritas] magnas 30 qua] quam *ei] e 32 lumina] tempora 36 quem] quam *Inter 38-9 dist. 133-4 extat, sed etiam geminatum post $132 \quad 48$ calfacti] calefacti $\mathbf{5 2}$ qui] quis *dissimulare] dissimilare 53-54 om. *55 Battiades inimicum devovet Ibin] Bachiades quondam devoverat ybim *64 atra] acta *65 haectibi] hoc mihi $\quad$ *68 cum Iove] cum Iane 76 netis] nectis *79 angue] agne *apillis] capellis $\mathbf{8 4}$ adeste Chao] adesse thori "85 fido dira] dira fido canuntur] canantur 86 peragunt] peragant *89 ut non] non ut *dicta] vota 90 Pasiphaes] phasiphes 91 quasque] quas ${ }^{* 93}$ fictum] fixum 94 commoveantve] commoveantque $96 \mathrm{se]} \mathrm{sa(err.} \mathrm{man.)}$ 98-99 ades] adest 101 ominibusque] hominibusque *laevis] undis *105 votis] votas *106 meis] tuis *107 amnis tibi] animas 109 sit] om. 111 tibi] om. 117 semper] om. nec] ne "119 putere] puteres *Post 119 vv. 122, 121, 120 hoc ordine *124 optatam fugiat] optatumque fuget *125 cruciatos spiritus artus] cruciatus spiritus artu 127 Evenient] et venient 130 mortis... semper] semper... mortis Inter $v \boldsymbol{v} .130-1$ dist. $130 a b$ extat $t^{19}$ *dist. 133-4 sequitur post 132, sed etiam post 38 *133 unquam] nunquam *139 nec] ne *finiet] finiat 140 in manes] inmanes 141 tunc] tu 142 exanguis] exanimis Dist.143-4 om. *149 peregrinae] peregrina 150 sive] inque *151 dignatus erit] erit dignatus 153 quidquid] quicquid *157 ante os] animos 159 colubrae] colubris 164 proiciere] proiecere "168 respuet] respinet *169 unguibus et] unguibusque et trahet] om. *175 petensque] patensque 178 Aegypti] egisti Post 180 dist. 181-182 sequitur 182 praebet] prestat ${ }^{*} 185$ sectos] sertos *186 tertia] tertius ${ }^{*} 192$ nunc] et *195 poenas] penna *197 canam] caue ab Ida] in hibla 198 aut] et *mare] mari *199 nascuntur] nascitur 202 fiat] fiet 205 venient] veniant *207 lacrimae] lacrimis 210 stella levisve] stellaque clara 211 non] nec *214 Maia] milla 215 nec quicquam] ne quicquam *216 falciferique] saltiferique *219 haec] hoc gravis] om. Allia] alia *227 unxerunt] iunxerunt 229 inbuerunt] imbuerant ${ }^{*} 233$ tinctis ferrugine pannis] pannis stigine tinctis 237 stipite] cespite *238 amorunt] ad mortem ${ }^{*} 240 \mathrm{sic}$ sit *una] vana 241 movimus] vovimus *244 nevit] venit *infesta] infestas *pulla] pilla 245 longa] longua 246 fata] facta *247 ille] hic ex me] et me ${ }^{*} 249$ pondera] carmina 253 clavigeri] claugeri *254 geras] genas 256 armatique] armatusque vulnus inermis opem]

${ }^{18}$ Sed huiuscemodi ineptias et discrepantias ortographicas posthac non adnotabo.

${ }^{19}$ finiet illa dies que te mihi subtrahet olim / finiet illa dies que tibi sera venit. 
vulnus inherme pontens 257 in Aleia decidit arva] aliena indecidit arva 258 paene] pena *259 Amyntorides] amantorides *262 uterque] utraque 265 ille] ipse *268 quos] quod 269 Aetnaeus] ethneneus 270 Teolemus] theolenus $\left.{ }^{*} 273 \mathrm{ut}\right]$ ubi ${ }^{*} 275$ tumidis melior Neptunus] tumidis neptunnus melior *276 quam] et cui] qui *278 ratis] rati 281 turpe] turpo *284 Hercei] crethei 285 a e $* 289$ fata] facta 291 inpune] inpugne 292 fixus alas] pasce tuo 293 Echecratides] eregratides 294 proiciare] praecipitare 299 Achaei] athei 301 Achillidem] achileidem 302 tegula] ragula *303 quiescant] regebant 306 cereri] celeri *dissimulare] dissimilare *307 utque] atque *308 Cantharidum] tassalidum sucos] succos *309 sicut] ut sic *313 Iovis] iovi parantes] parentes *314 condat] cum dat *315 necatorum] vecatorum 316 subsidens] succendens *317 Sycione] scicione 319 insutus pelle iuvenci] inductus pelle iumenti *321 Pheraei] philei *322 leto] lecto *323 ut] non Aleuas] alebas 324 experiare] experiere *325 utque] atque *327 quaeque] quodque *Phialesia] philea *328 venerunt] veniunt 329 Amastriacis] amateratis Lenaeus] lempneus 331 Eurydamas] euridamus *333 qui quae] quiqui *335 passa... genus] genus... passa *336 Actea] athea Post 338 disticha 637-8, 439-40, 461-2 sequuntur 339 scopulus] scopulis *340 Euboico] embeico 344 qui] cui

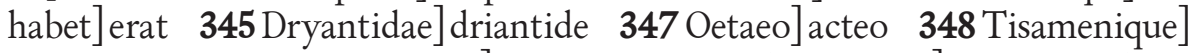
thisimanique Callirhoesque] caliganesque *351 iunxit] vinxit *352 ancillae] anchille *nece] vere Post 352 disticha 353-4, 355-6 sequuntur *355 letum] lectum *358 per crimen] termine 359 Pelopea] polopeia Thyestae] tiesty *360 Nyctimeneque] victimeneque 361 neue magis pia sit capitique parentis amica] filia neue magis capiti sit fida parentis 362-3 om. *365 quorum fastigia vultus] proiecta cadavera quorum (quarum post correctionem) 366 Bracchia] oraque *372 facta] rapta *375-6 altum... rogum] altum... regum 377 obscuri] obscuris oris] horis *378 Sphinga] spina 380 quod] quos $\quad 382$ suis] meis 383 Therodamanteos] therodomantheos *385 vorax] verax 387 demisit] dimisit 388 ut] aut 391 sex bis ut] et velut famulae] famuli *394 cecidisset] cecidissi *395 ut] et 396 Lemnia] lennia 397 annum] longum *399 Antaei] antea *403 vindice] vertice 404 Nessus] nessi $406 \mathrm{ab}$ ipse] ab urbe 407 Sinis] cinos Sciron]siron Polypemone] polihemane 408 parte] om. post sui *iuvencus] iuventus *409 pressas] pransas *410 adspiciens] aspectans *411 laeto vidit] vidit lecto $\quad 412$ Cercyonea] gerionea 413 quem] que $\quad 414$ evenient] eveniant *aut his] iustis *415 Sicula] sucula Aetna] hora *416 vidit] venit 418 pontem] pontum 419 et] ut ${ }^{*} 420$ Destituatque... petitus opes] destituasque... petitas oves *422 subtrahitur] subiceretur pedi] pede 426 conficiare] destituare $\quad * 27$ quaque] quacumque 429 Solis] rursus *ortus] ortum 430 rursus] solis *431 repetes] reperent *432 cibi fallere] tibi fasse *434 tu Teleique] tindutique *437 aere] ore imitere] imitare Post 438 dist. 439-40 sequitur *439 Phalaris] fallens ense] ore *443 
aut] at caeni] scevi *445 atque] ac 446 iactis] sparsis 447 Pytheides] pentides Medusae] meluse *448 eveniant] et venient 450 proiecta] coniecta *451 totque] que tot ${ }^{*} \mathbf{4 5 2}$ inferiis] inferis culter] cultor ${ }^{*} \mathbf{4 5 3}$ Cybeleia] tibeia $\mathbf{4 5 5}$ nec femina nec vir] nec vir nec femina *Attis] ante 457 subito Magnae] magnae subito *458 ut est celeri victaque] et ut sceleri victima Dist. 461-2 in secuentia 338, 637-8, 439-40, 461-2, 339 habet, sed abest post $460 \quad{ }^{*} 463$ Abantiades] habent hiades ${ }^{*}$ Cycneius heros] lingneus heror 466 Theudotus] theodorus *469 telo feriare] tela ferialem 470 Hipponoo] hipomeo *Dexitheaeque] dexiceesque *471 Autonoes] atonees Maia] mala 472 ut] et 474 Arctos] artos *475 Macelo] macolo *rapidis icta est] iacta est rapidis *477 sis] sit *Delos] delon *482 Oeagri] oleagri $\mathbf{4 8 3}$ Hypsipyles] ysiphiles $\quad{ }^{*} \mathbf{4 8 5}$ cautius altos] calcius equos 488 iuvit] iuit *489 suo] fuit *antro] alto *490 Cacus] captus 91 Nesseo tincta] nesse otinta $\quad{ }^{*} 492$ Euboicasque] emboicasque 494 socraticum] sacraticum *496 ut iliaca] et ylianta *497 eadem] eademque 499 Lindia] lidia 500 quae] quod $\mathbf{5 0 2}$ Phalaeceae]phiphegee necis] lucis ${ }^{\text {ac }}$ *503 Lycurgidem] lignigidem *504 Idmonaque] idmona *rumpat] rumpit $\quad$ 506 suis] focis 507 sive] atque *508 Phryx ac] phryxa *Berecyntiades] bene cinthiades *509 contigerit] contingerit *510 Corcyraeum] cor circum Cressia] tregia 511 Aleuae] alebe 512 Leoprepidae] letoride cum] cui 513 Euenus] ebenus $\mathbf{5 1 5}$ Astacidaeque] irtaquideque defixa] decisa 516 hominis] homini *sit] sic *518 cremanda pyrae] cremanta pire $\mathbf{5 2 3}$ Athenin] athenas $\quad{ }^{*} \mathbf{5 2 5}$ fertur] feruntur $\mathbf{5 2 6}$ exitii] excicii *527 Oresti] oreste $\quad 530-1 \mathrm{om} . \quad * 532$ haereat] lateat *533 lacer] latens silva] silvis $* 537$ invita] invicta sororis] sorori 539 conditor] cognitor *Myrrhae] terre $\mathbf{5 4 0}$ urbis] orbis *541 Inque tuis] utque tui Achaeo] acheuo *542 condat] credat 543 saxis] flammis *546 eas] esis 548 om. 549 utve] utque Syracosio] siracusio *praestricta] prescripta *550 via] vie $\mathbf{5 5 1}$ nudave] nudaque derepta] direpta $\mathbf{5 5 2}$ Phrygium] frigii flumen] fulmen *553 Medusae] me duce *555 Potniadum] pomeadum *morsus ... Glaucus] glaucus... morsus *equarum] aquarum *556 salias] filias 558 Gnosia] noxia 559 Anyti] veluti ${ }^{*} 561$ cedat] cadat $\quad$ *563 videas quod] videras qui $\mathbf{5 6 4}$ in urbe] ab arce $\mathbf{5 6 9}$ loquax] locax guttur] guture acerno] agenor $\quad{ }^{*} \mathbf{5 7 0}$ pollice vocis iter] vocis iter pollice $\left.\quad 575 \mathrm{ea}\right] \mathrm{om}$. dextra] dextera $\mathbf{5 7 7}$ moriturus] moribundus $\quad * \mathbf{5 7 8}$ equis] aquis $\quad \mathbf{5 7 9 - 6 2 6}$ om. $\quad$ *627 quos fortis] fortis quos *628 eat] erat $\quad{ }^{6} 629$ quiescas] quiescat *630 comites] comes tum] tu *necis] meus $\mathbf{6 3 1}$ quam] et *morti] morte *Rhamnete] favente $\mathbf{6 3 3 - 4} \mathrm{om} . \quad 635$ utque] utve Dist. 637-8 post 338, sed etiam hic *637 inter Geticasque] interiectasque 639 tibi] om. * *subito] subdito *640 inmemores] inmemor es $\quad$ *641 di] dum 643 et] sed 
